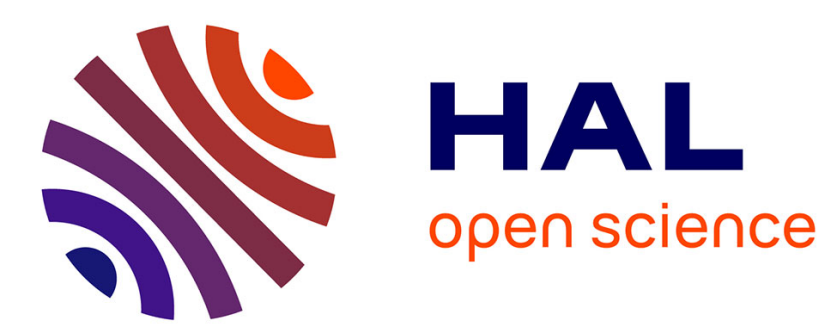

\title{
Depinning of evaporating liquid films in square capillary tubes: Influence of corners' roundedness
}

Fabien Chauvet, Paul Duru, Marc Prat

\section{To cite this version:}

Fabien Chauvet, Paul Duru, Marc Prat. Depinning of evaporating liquid films in square capillary tubes: Influence of corners' roundedness. Physics of Fluids, 2010, 22 (11), pp.112113. 10.1063/1.3503925 . hal-01360012

\section{HAL Id: hal-01360012 \\ https://hal.science/hal-01360012}

Submitted on 5 Sep 2016

HAL is a multi-disciplinary open access archive for the deposit and dissemination of scientific research documents, whether they are published or not. The documents may come from teaching and research institutions in France or abroad, or from public or private research centers.
L'archive ouverte pluridisciplinaire HAL, est destinée au dépôt et à la diffusion de documents scientifiques de niveau recherche, publiés ou non, émanant des établissements d'enseignement et de recherche français ou étrangers, des laboratoires publics ou privés. 


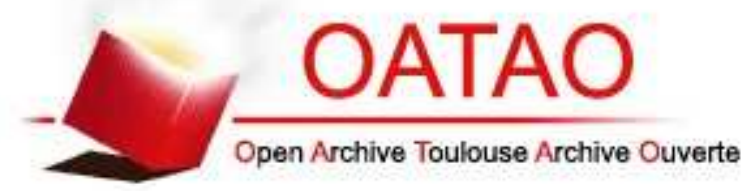

\section{Open Archive TOULOUSE Archive Ouverte (OATAO)}

OATAO is an open access repository that collects the work of Toulouse researchers and makes it freely available over the web where possible.

This is an author-deposited version published in : http://oatao.univ-toulouse.fr/ Eprints ID : 16043

To link to this article : DOI : $10.1063 / 1.3503925$

URL : http://dx.doi.org/10.1063/1.3503925

To cite this version : Chauvet, Fabien and Duru, Paul and Prat, Marc Depinning of evaporating liquid films in square capillary tubes:

Influence of corners' roundedness. (2010) Physics of Fluids, vol. 22 (n० 11). pp. 112113-1-112113-14. ISSN 1070-6631

Any correspondence concerning this service should be sent to the repository administrator: staff-oatao@ listes-diff.inp-toulouse.fr 


\title{
Depinning of evaporating liquid films in square capillary tubes: Influence of corners' roundedness
}

\author{
F. Chauvet, P. Duru, ${ }^{\text {a) }}$ and M. Prat \\ Université de Toulouse, INPT, UPS, IMFT (Institut de Mécanique des Fluides de Toulouse), \\ Allée Camille Soula, F-31400 Toulouse, France and CNRS, IMFT, F-31400 Toulouse, France
}

\begin{abstract}
In this paper, evaporation of a volatile, perfectly wetting liquid confined in an initially filled capillary tube of square internal cross section is studied, when conditions are such that liquid films develop along the tube internal corners under the effect of capillary forces, as the bulk meniscus recedes inside the tube. More precisely, the emphasis is on the moment when the liquid film tips depin from the tube top once they have reached a critical length, a phenomenon observed in experiments. A model taking into account liquid corner flow and phase change at the film tip is proposed in order to predict the critical film length at depinning. The model is found to be in good agreement with experimental data and highlights that the critical film length depends strongly on the degree of roundedness of the tube internal corners. Thus, it is crucial to take into account this purely geometrical factor when modeling evaporation in polygonal capillary tubes or, more generally, corner flows in a rounded wedge.

doi:10.1063/1.3503925
\end{abstract}

\section{INTRODUCTION}

Evaporation from liquid menisci in confined environment is of importance in many applications, such as cooling technologies ${ }^{1}$ or drying of porous media. ${ }^{2}$ Understanding the fundamental processes involved in these applications is essential and has motivated many studies (see, for example, Ref. 3 and references therein). In this context, the consideration of evaporation in a single tube or microchannel is an important step for the understanding of more complex systems, such as, for example, networks of interconnected capillaries, which are common in microfluidic applications ${ }^{4}$ and which are also frequently used as conceptual models of porous media. ${ }^{2}$ Our motivation for the consideration of such an elementary system originates more specifically from experimental results in etched networks of channels of rectangular cross section, showing that evaporation was much faster than initially expected. ${ }^{5}$ The effect was conjectured to be due to the effects of liquid films trapped by capillarity in the corners of the channels invaded by the gas phase (see Fig. 1). Since then, the consideration of these corner films in relation with drying problems has motivated several modeling and numerical studies ${ }^{6,7}$ and it is now widely admitted that these films play a major role not only in etched networks, but also in most porous media. However, this is only very recently that careful experimental studies were developed with the twofold objective of gaining a better understanding of evaporation in the presence of corner films and developing quantitative predictive models. ${ }^{8,9}$ The present paper notably highlights the influence of corner roundedness. As we shall see, the tube evaporation kinetics is greatly affected by the tube degree of roundedness. Hence, a somewhat tiny geometrical detail has a great influence on evaporation. This has impor-

\footnotetext{
a) Author to whom correspondence should be addressed. Telephone: +33 534-32-28-77. Fax: +33-534-32-28-99. Electronic mail: duru@imft.fr.
}

tant implications for the drying of porous media since the study clearly suggests that evaporation in porous media should depend on tiny details of the pore space geometry and therefore contributes to explaining why quantitatively predicting the evaporation rate of porous media remains a challenging problem. Also, it is interesting to notice that the mass transfer driven evaporation from a circular tube is well understood since the 19 th century ${ }^{10}$ and is a classic textbook problem, usually referred to as the "Stefan tube problem." The situation is completely different in tubes of polygonal cross section. The presence of corners and associated corner liquid films leads to much greater evaporation rates $^{9}$ but makes the analysis significantly more involved. The evaporation enhancement is explained by the corner liquid films. As sketched in Fig. 1, "thick" and elongated liquid films are trapped by capillary forces along the tube's four internal corners in the square tube considered in the present study, if the liquid wetting contact angle $\theta$ is inferior to a critical contact angle $\theta_{c}=45^{\circ} \cdot{ }^{12,13}$ These corner films provide transport paths for the liquid between the receding bulk meniscus and the entrance of the tube. The liquid is transported within the films under the action of the pressure gradient induced by the meniscus curvature variation along the films, up to the tube's opened end, where it evaporates. Viscous resistance to the corner flow and gravity (when the tube is held vertically) tend to oppose to the capillary pumping. The relative importance of these three effects can be quantified by introducing two dimensionless numbers: the capillary $(\mathrm{Ca}=$ viscous effects/capillary effects) and the Bond numbers (Bo $=$ gravity effects/capillary effects). When there are no corner films $\left(\theta \geq \theta_{c}\right)$, the phase change takes place at the bulk meniscus and vapor is transported by molecular diffusion up to the tube top as in the Stefan tube problem (tube of circular cross section).

Corner liquid films are, of course, important not only for mass transfer driven evaporation problem. Situations similar 


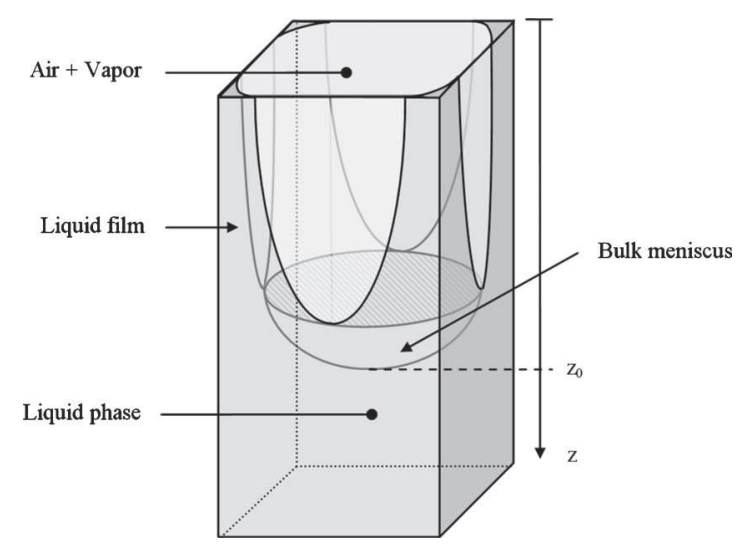

FIG. 1. Sketch of the thick liquid films in a capillary tube of square cross section. The bulk meniscus position is denoted as $z_{0}$.

to the one described above, where a liquid confined in a corner or wedge flows under the action of capillary forces, are encountered in various research fields and have been discussed widely in the literature. For instance, the imbibition of a wetting liquid along a corner can be related to oil recovery problems in a porous medium. ${ }^{14,15}$ Corner flows are also relevant in many applications involving microsystems, such as microheat pipes. In a microheat pipe, a volatile liquid is transported by capillary pumping in wedges to some hot regions of the device where it vaporizes. In contrast with the situation considered in the present paper, evaporation is driven by heat transfer in this case. Numerous studies focused on the length of the liquid-wetted region, an important parameter to model to quantify the microheat pipe efficiency and cooling capabilities ${ }^{1,16}$ and on the Marangoni flows resulting from the temperature gradients, which can oppose the capillary-induced motion, leading to stationary interface shape. ${ }^{17-19}$ The modeling of corner flows in a wedge usually relies on the assumption of a perfectly sharp corner in the wedge. If this is certainly relevant when dealing with microsystems made using Silicium-etching based techniques, which lead to sharp angles down to submicron scale, this is not the case when working with corners fabricated from solid materials by other techniques, such as machining or extrusion (as is the case for glass capillary tubes of polygonal cross section). However, we are aware of only a few works that tried to incorporate the corner or wedge degree of roundedness in the description of the abovementioned systems. Several works focused on the variation of the viscous resistance for corner flow as a function of the wedge degree of roundedness. ${ }^{14,20,21}$ All these works show that the resistance is increasing with the degree of roundedness and can then be much larger than that for the sharp corner case. Dong and Chatzis ${ }^{15}$ have then shown that the spreading dynamics of a wetting droplet in a rounded wedge is going to be slowed down compared to the sharp corner case. In Chen et al. ${ }^{21}$ the authors mention that the tube roundedness is also going to limit the drop final extension in the wedge, in the case of a perfectly wetting fluid, which would spread to infinity in a sharp wedge in the absence of gravity. It can also be mentioned that in a static case, in the absence of gravity, it has been shown by Concus and Finn ${ }^{22}$ that the classical condi- tions established in previous works ${ }^{12,13}$ for corners films to develop- $\theta<\theta_{c}=\pi / 2-\alpha$, where $\alpha$ is the corner halfangle-is modified when the corner is rounded. For a given $\alpha$, when the degree of roundedness is nonzero, smaller critical contact angles have to be considered, compared to the perfect corner case, for corners films to be present.

As mentioned before, the main scope of this paper is to study how evaporation in a square capillary tube is affected by the tube degree of roundedness. We deal with slow evaporation in a stagnant dry air, under atmospheric pressure, and at ambient temperature. Under these conditions, the phase change is limited by mass transfer and the system is supposed to be isothermal. Experiments using an infrared thermography technique have confirmed the fact that the phase change takes place at the top of the square capillary tube. However, this is true only for a first stage of the experiment. Indeed, it was shown by infrared thermography ${ }^{9}$ and by charge-coupled device (CCD) camera visualizations ${ }^{23}$ that the corner films depin from the tube top after a while and then recede inside the tube, a phenomenon not predicted by earlier modeling attempts. ${ }^{9}$ More precisely, the drying kinetics of a square tube was described in Chauvet et al. ${ }^{8}$ and shown to be characterized by three main periods: a first period where the films remain attached to the tube entrance and slowly get thinner in the tube entrance region (as well as within the tube), and two periods where the films' tip is located inside the tube. The evaporation flux is much higher during the first period, which essentially depends on the external mass transfer (as opposed to the third one, which is dominated by the transfers inside the tube and the second one, which depends on both the external and internal transfers). The event marking the end of the first period is the films' depinning from the tube entrance. Since the first period is the high evaporation flux period, it is particularly important to predict its duration and therefore the films' depinning. Consequently, the main goal of the present paper is to predict the depinning through an improved modeling of the films' thickness evolution during the first period and at depinning, notably by taking into account the effect of tube roundedness and then to compare the model predictions to some experimental results.

The paper is organized as follows. First, we rapidly describe the experimental setup and techniques used. Then, we first show how the internal corner degree of roundedness is controlling the maximal extension of hydrostatic, nonvolatile corner liquid films before turning to the more involved case of an evaporating fluid in Sec. IV. The predictions of the model are then compared to experimental data in Sec. V. Conclusions are drawn in Sec. VI.

\section{EXPERIMENTAL SETUP}

The experimental setup consists of a capillary tube, held vertically and glued at one of its ends by an epoxy resin directly to a syringe tip, the other end being opened and placed in stagnant dry air. The tube filling is controlled by a precision syringe pump (PHD 2000, Harvard Apparatus).

Two types of capillary glass tubes were used in the present work. First, a $10 \mathrm{~cm}$ long square capillary tubes 
TABLE I. Capillary tubes characteristics. The degree of roundedness was obtained by averaging the radii values of the best-fitting quarters of circular arcs to the internal corners of several tube cross sections, obtained by cutting a tube with a precision saw. The uncertainty on the degree of roundedness is the standard deviation to the mean value.

\begin{tabular}{lcccc}
\hline \hline & Tube 1 & Tube 2 & Tube 3 & Tube 4 \\
\hline Supplier & Vitrocom & Vitrocom & Hilgenberg & Hilgenberg \\
Internal dimension $d(\mathrm{~mm})$ & 1 & 0.4 & 0.95 & 0.49 \\
Tube wall thickness $(\mu \mathrm{m})$ & 200 & 200 & 108 & 55 \\
Degree of roundedness $r_{0}(\mu \mathrm{m})$ & $105 \pm 2.5$ & $32 \pm 1.5$ & $48 \pm 5$ & $20.5 \pm 1.5$ \\
\hline \hline
\end{tabular}

made of borosilicate glass and supplied by Vitrocom. The internal side length and wall thickness of the tubes are given in Table I. As can be noticed by imaging tubes' cross sections with an optical microscope, the tubes' internal corners are not sharp. The degree of roundedness $r_{0}$, as defined in Fig. 2(a), is estimated by fitting the internal corners' shapes by quarters of circular arcs for several tube cross sections [see Fig. 2(b)]. The values of $r_{0}$ for the capillary tubes used are given in Table I. Second, a $10 \mathrm{~cm}$ long square capillary tubes made of borosilicate glass and supplied by Hilgenberg. The internal side length and wall thickness of the tubes are given in Table I. As for the Vitrocom capillary tubes, the tubes' internal corners are not sharp. However, the internal corner cross section shape does not consist of a circular arc contrary to the Vitrocom tubes, probably due to a different fabrication process. The degree of roundedness $r_{0}$ was nonetheless estimated as previously, by fitting the internal corners' shapes by quarters of circular arcs, for several tube cross sections, the quarters of the circular arc being tangent to both the tubes' lateral sides and the straight segment forming the angle [see Fig. 2(b)]. The corresponding values found for $r_{0}$ are given in Table I. Note that the values for $r_{0}$ are found to be smaller for this kind of tubes than those for the tubes supplied by Vitrocom. There is notably a factor of 2 between the two values for the $d \approx 1 \mathrm{~mm}$ tubes (tubes 1 and 3 ). Thus, these differences in the tube corners' degree of roundedness, depending on the supplier, allowed us to vary this parameter, which will prove to be a key parameter in the present study. Three perfectly wetting fluids were used: heptane, 2-propanol, and a nonvolatile silicone oil (47V5). The fluid properties relevant for this study are shown in Table II.

The measurements performed in the present study rely on video-imaging of the capillary tube, using an ombroscopy configuration in order to detect easily the location of the interface between the liquid and the gas. Two CCD cameras (Sensicam, PCO) were used. The first CCD camera is facing one side of the capillary tube and images are taken at low magnification (spatial resolution $\approx 13$ pixels $\mathrm{mm}^{-1}$ ). It is used to detect the bulk meniscus location $z_{0}$ (see Fig. 1). When the liquid films are present up to the tube opened end, this also amounts to measure the film length $L$, i.e., $L=z_{0}$.

The second CCD camera provides high magnification images of the tube top (spatial resolution $\approx 654$ pixels $\mathrm{mm}^{-1}$ ). The optical axis is aligned with one of the tube diagonals so that three corner films out of four are seen on the high magnifications images. The focus is made on the two lateral corner films. Image processing, based on optical geometry considerations, allows determining the film thickness $e=(\sqrt{2}-1)\left(R-r_{0}\right)$, as sketched in Fig. 2(a), made dimensionless by its value at the beginning of the experiments $e_{0}$ (see Refs. 23 and 24 for more details). The film thickness measurement is typically performed on one of the two lateral films imaged, at least one tube diameter away from the film tip, i.e., in a region where the film longitudinal curvature can be neglected (see Sec. III), which is a necessary condition for the present analysis to be correct. Also, the moment when the lateral films depin from the tube top can be determined by careful inspection of the recorded images. This is also possible for the liquid film visualized in the corner closest to the camera, despite the fact that it is blurred on the images because it is slightly off-focus. The image acquisition rate is typically $0.05 \mathrm{~Hz}$ (one image taken each $20 \mathrm{~s}$ ). For some experiments, it is found that the film tip depinning in each corner does not occur within the same time interval between two given recorded images: after the first film depinning, the following images can still show one or two corner films still present up to the tube top for a short

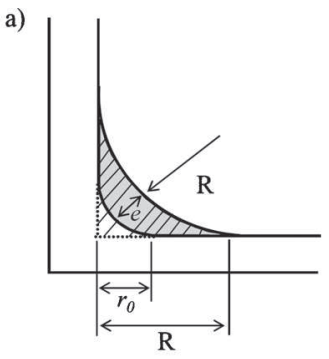

b)

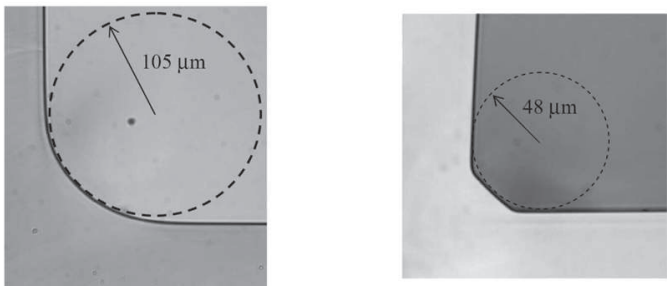

FIG. 2. (a) Radius of curvature $R$ and film thickness $e$ in the tube cross section plane at a location along the tube where the longitudinal curvature of the liquid-gas interface is negligible compared to the transverse one $(1 / R)$. The degree of roundedness is denoted as $r_{0}$. Note that the tube external corners are also rounded but as it does not play a role in the present study, it is not shown in this sketch. The hatched region is the union of the liquid and solid corners. Its area at a given vertical position $z$ is $A_{c} / 4$ [see Eq. (7) in Sec. IV]. (b) Roundedness of a square capillary tube internal corner, as visualized by an optical microscope with a $\times 20$ magnification; left: tube 1 , right: tube 3 , see Table I. 


\begin{tabular}{lccc}
\hline \hline & $47 \mathrm{~V} 5$ & Heptane & 2-Propanol \\
\hline Density $\rho_{l}\left(\mathrm{~g} \mathrm{~cm}^{-3}\right)$ & 0.910 & 0.679 & 0.781 \\
Surface tension $\gamma\left(\mathrm{mJ} \mathrm{m}^{-2}\right)$ & 19.70 & 19.66 & 20.93 \\
Liquid viscosity $\mu_{l}\left(\mathrm{~kg} \mathrm{~m}^{-1} \mathrm{~s}^{-1}\right)$ & 4.55 & 0.387 & 2.04 \\
Equilibrium vapor mass concentration $c_{e}\left(\mathrm{~kg} \mathrm{~m}^{-3}\right)$ & $\ldots$ & 0.230 & 0.134 \\
Vapor-air diffusion coefficient $D\left(\mathrm{~m}^{2} \mathrm{~s}^{-1}\right)$ & $\ldots$ & $7.23 \times 10^{-6}$ & $9.70 \times 10^{-6}$ \\
\hline \hline
\end{tabular}

time duration. This situation will be referred to as "differential depinning" in the following (see Sec. V). However, the difference between the longest corner film imaged (found for the latest depinning) and the shortest is always small, being at most one tube internal diameter. More details on the experimental setup and techniques can be found elsewhere. 9,23,24

\section{ROUNDNESS-LIMITED EXTENSION OF A HYDROSTATIC LIQUID FILM}

To highlight the effect of the tube internal corners' degree of roundedness on the corner film extension, we first focus on the simple case of a nonvolatile liquid at rest, confined in a square capillary tube. The liquid is perfectly wetting so that thick liquid films develop along the internal tube corners, as sketched in Fig. 1. The variation of the pressure in the liquid film $p_{l}$ along the vertical direction $z$ is given by the equation of fluid statics

$$
\frac{d p_{l}}{d z}=\rho_{l} g \text { for } 0<z<z_{0}
$$

where $\rho_{l}$ is the liquid density and $g$ is the gravity acceleration. At a given $z$, the liquid pressure is related to the gas ambient pressure $p_{a}$ by Laplace's law

$$
p_{l}(z)=p_{a}-\frac{\gamma}{R(z)} \quad \text { for } \quad 0<z<z_{0},
$$

where $\gamma$ is the surface tension and $R(z)$ is the curvature radius of the liquid-gas interface in the plane perpendicular to the tube axis [see Fig. 2(b)]. Note that the longitudinal curvature, i.e., the curvature along the tube axial direction $z$, is neglected in this simple model, which is a usual assumption in the corner flow literature. Recently, Yang and Homsy ${ }^{17}$ have studied the case of a liquid meniscus in a V-shaped wedge under an imposed axial temperature gradient. The equilibrium meniscus shape is fixed by the competition between the Marangoni stress and the capillary pressure gradient. They have shown that to neglect the longitudinal curvature is justified over the full film extension by deriving an expression for the capillary pressure drop and showing that it is dominated everywhere by the transverse curvature term compared to the axial one (note that this remains true as long as the sum of the contact angle and of the corner half-angle is not $\pi / 2$, which is the case in the present study, where $\theta \approx 0$ and the half-angle is $\pi / 4)$. This is also true when the liquid film has dried out, due to the heat flux imposed on the wedge. However, we believe that the situation is different when the liquid films are "pinned" to the three-dimensional geometric singularity consisting of the junction between the tube internal corner and the tube top external surface, at the tube entrance. Consequently, we cannot strictly make use of Yang and Homsy's result to argue that the longitudinal curvature is negligible when compared to the transverse one, over the full film extension. However, the longitudinal curvature affects the shape of the elongated films only very close to their tips in the present case, as can be checked on high magnification images of the film tips. Clearly, if the longitudinal curvature were to be non-negligible compared to the transverse one $[1 / R(z)]$, it would be in this region of the films, which is of a very limited spatial extension and small compared to the total film length $L(>10 d$ in the present experiments). Thus, it can be expected that to take into account the longitudinal curvature would result only in a marginal correction to the case where only the transverse curvature is considered.

As the hydrostatic pressure drop over the vertical extension of the bulk meniscus is negligible compared to the capillary pressure jump at the bulk meniscus, the total curvature of the meniscus can be taken as the purely capillary solution for the meniscus curvature 25,26

$$
R_{b m}=\frac{d}{2 \chi}
$$

where $\chi$ is a dimensionless curvature, which is a function of both the contact angle and the critical contact angle above which no corner film exist. For a perfectly wetting liquid in a square capillary tube, $2 \chi \approx 3.77$. In the present modeling, it is assumed that the purely transverse radius of curvature of the corner films tends toward $R_{b m}$ when the corner films match to the bulk meniscus, i.e., when $z \rightarrow z_{0}$. As mentioned in Sec. II, the film length $L$ is obtained experimentally by measuring the bulk meniscus bottom position $z_{0}$. Consequently, directly comparing such experimental data to predictions of film lengths from the model comes down to neglecting the vertical extension of the bulk meniscus compared to the film length. This is reasonable for the elongated films of interest in the present study.

Combining Eqs. (1) and (2) leads to the following expression for the radius of curvature evolution with $z$ :

$$
R^{*}\left(z^{*}\right)=\frac{1}{1+\mathrm{Bo}\left(1-z^{*}\right) / \epsilon} .
$$

In this expression, $R^{*}\left(z^{*}\right)$ is the radius of curvature made dimensionless by $R_{b m}$ and $z^{*}=z / L$. The Bond number Bo is defined as $\rho_{l} g R_{b m}^{2} / \gamma$ and $\epsilon=R_{b m} / L$. For a perfectly sharp corner, Eq. (4) predicts an infinite maximal length for the 
corner film: $L_{\max } \rightarrow+\infty$ when $R^{*} \rightarrow 0$ at $z^{*}=0$. In fact, the above description breaks down when the film thickness becomes so small that disjoining pressure effects begin to act. Taking a typical cutoff thickness for that transition of $\approx 10 \mathrm{~nm}$, Eq. (4) still leads to a remarkable length $L_{\max }$ of $\approx \mathrm{O}\left(10^{6}\right) \mathrm{m}$ for $47 \mathrm{~V} 5$ oil in a $d=1 \mathrm{~mm}$ square capillary tube $(\mathrm{Bo}=0.032)$. Actually, the maximal film length is drastically limited by the tube internal corners' degree of roundedness which provides a lower limit for the film curvature radius $R$, as mentioned qualitatively by Bico and Quéré ${ }^{27}$ (note that Ramos and Cerro ${ }^{28}$ also deduced from this remark a way to measure the very small contact angle of almost perfectly wetting liquid on glass). The maximal film length is then obtained when $R^{*} \rightarrow r_{0}$ at $z^{*}=0$ and reads

$$
L_{\max }=\frac{R_{b m}}{\mathrm{Bo}}\left(\frac{1}{r_{0}^{*}}-1\right),
$$

where $r_{0}^{*}=r_{0} / R_{b m}$. One then obtains $L_{\max } \approx 12.65 \mathrm{~mm}$ using $47 \mathrm{~V} 5$ oil properties in tube $1\left(d=1 \mathrm{~mm}, r_{0}^{*}=0.396\right)$. Thus, the length of a static corner liquid film depends strongly on the degree of roundedness of the tube internal corners. Note that the above description breaks down when the roundedness of the tube is such that the film length is no longer large enough compared to $d$ so that the longitudinal curvature has to be taken into account.

Experiments performed with 47V5 oil, a perfectly wetting, nonvolatile fluid, allowed to test the predictions of the above analysis. Note that in these experiments, the tube was first filled completely with silicone oil, which was then drained using the syringe pump. Several drainage steps were realized successively in order to impose several liquid film lengths. For each bulk meniscus position $z_{0}$, a film thickness measurement was performed, a long time (30 $\mathrm{min})$ after the previous drainage step, to allow the films to relax to their hydrostatic shape. In Figs. 3(a) and 3(b), respectively, the measured film thickness $e$, made dimensionless by $e_{0}$, the film thickness measured after the first drainage step, is shown as a function of the imposed bulk meniscus position $z_{0}$ for tubes 1 and 2, respectively. It is important to note that the film thickness measurements were performed at least one tube diameter away from the tube top: at $z_{m}=1 \mathrm{~mm}$ for tube 1 and at $z_{m}=0.67 \mathrm{~mm}$ for tube 2 . These measurements points were chosen arbitrarily sufficiently "far" from the very top of the tube, where some longitudinal curvature effects are visible, as explained in Sec. II. It was checked that the results do not vary significantly with the exact location where the image processing is performed. For $z_{0}<L_{\max }$, the theoretical predictions concerning the film thickness, shown as solid lines in Fig. 3, have been obtained using Eq. (4) with $L=z_{0}$ and $z^{*}=z_{m} / z_{0}$. Theoretically, when the bulk meniscus position reaches the value $L_{\max }$ given by Eq. (5), the films are supposed to depin from the tube top. However, the film thickness at the measurement point is not zero at that moment. For $z_{0}>L_{\max }$, the theoretical predictions shown in Fig. 3 were obtained by assuming that once a film has depinned, its shape does not evolve and is simply translated further down the tube as the bulk meniscus recedes. Consequently, theoretical predictions shown in Fig. 3 are nonzero up to $z_{0}$ values equal to $L_{\max }+z_{m}$. The agreement between the experiments and the theoretical prediction concerning the film thickness evolution at the measurement point is good. In particular, the maximal film extension is well predicted as the thickness is found to go to zero when $z_{0} \approx L_{\max }+z_{m}$. Note that the theoretical predictions for $r_{0} \pm$ the standard deviation to the average $r_{0}$ value (see Table I) have also been plotted in Fig. 3 as dotted lines to take into account the uncertainty on the $r_{0}$ value when comparing the theoretical predictions to the experimental data.

This simple hydrostatic case highlights the fact that the tube corners' degree of roundedness is a major parameter to predict the hydrostatic liquid film extension in a square capillary tube partially filled with a nonvolatile liquid. In the remainder of this paper, it will be shown that the tube corners' degree of roundedness is also a key feature in a much more complex situation, namely, when the confined liquid is evaporating.

\section{EVAPORATING LIQUID FILM: MODELING}

In this section, a model of evaporation in a capillary tube of square cross section is introduced, the results of which will be compared to experimental results in Sec. V. It is based on the earlier modeling attempts mentioned in Sec. I. The new key feature introduced here is that the present approach allows taking into account the corner internal degree of roundedness. As it will be seen in Sec. V, this is a crucial parameter to consider in order to get quantitative predictions from the model.

\section{A. Modeling and numerical technique}

Slow evaporation in stagnant, dry air is considered. Phase change is controlled and limited by vapor diffusion in the surrounding air and the system is assumed to be isothermal, i.e., the temperature variations due to the evaporation cooling effect are neglected. This assumption is supported by experimental data. Using an infrared thermography technique, ${ }^{9}$ the cooling induced by evaporation can be measured. The cooling is located at the top of the tube, where the phase change takes place until the films depin. The cooling is at most $2.5 \mathrm{~K}$ when using 2-propanol in tube 1 and less than $1.5 \mathrm{~K}$ for three others cases ${ }^{24}$ (2-propanol in tube 2 and heptane in tubes 1 and 2). These amplitudes of cooling are sufficiently small to consider the system to be isothermal, in the range of evaporation rates obtained in the present study. The starting point of the modeling follows the classical analysis of Ransohoff and Radke ${ }^{20}$ for corner flows, relating the liquid flow rate in the liquid films to the liquid pressure gradient by a Poiseuille-like law corrected for gravity

$$
q_{\text {liq }}(z)=-\rho_{l} \frac{A_{c} R^{2}}{\beta \mu_{l}}\left(\frac{d p_{l}}{d z}-\rho_{l} g\right) \quad \text { with } \quad 0<z<z_{0},
$$

where $\mu_{l}$ is the liquid viscosity and $A_{c}$ is the sum of the areas of the four liquid corner films and of the four "solid" corners, in a cross section at position $z$ [see Fig. 2(a)] 
a )

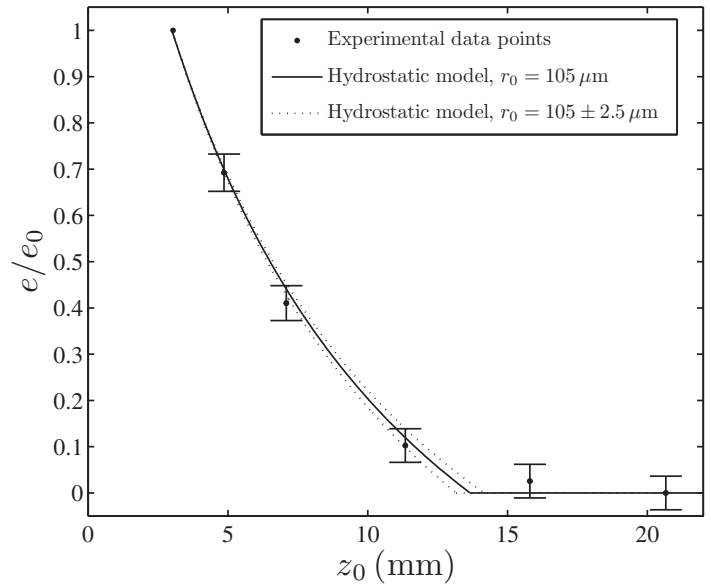

b )

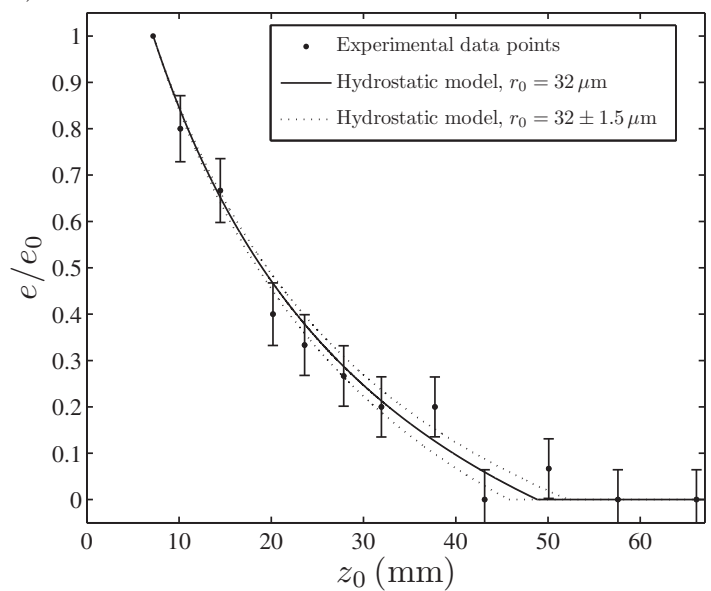

FIG. 3. (a) Dimensionless liquid film thickness $e / e_{0}$ as a function of bulk meniscus position $z_{0}$ for (a) tube 1 and (b) tube 2. Equation (5) gives $L_{\max }=12.65 \mathrm{~mm}$ for tube 1 and $L_{\max }=48.2 \mathrm{~mm}$ for tube 2 .

$$
A_{c}=\lambda R^{2},
$$

with $\lambda=4-\pi$, for a perfectly wetting liquid in a square cross section tube. ${ }^{14,25}$ In Eq. (6), $\beta$ is a dimensionless flow resistance depending on the tube corner shape, the contact angle, and the boundary condition at the liquid-air interface. In the present study, the shear stress on the liquid-vapor interface of the films can be neglected because the liquid viscosity is much larger than that of air. As in Sec. III, the liquid is considered as perfectly wetting on the internal tube wall. The dimensionless flow resistance for a rounded corner was obtained in three different studies. Ransohoff et al. ${ }^{20}$ solved the corner flow equation numerically using a finite element method and obtained a discrete set of values for the dimensionless flow resistance, depending on the contact angle, the shear stress on the liquid-vapor interface, and the degree of roundedness. An analytical formula is proposed in Zhou et al. ${ }^{14}$ using two classical approaches: the hydraulic diameter approach and thin film flow theory. In the two aforementioned studies, the dimensionless flow resistance, which is denoted $\beta$, is unbounded and diverge when $r_{0} / R \rightarrow 1$. A third approach, initiated by Ayyaswamy et al. ${ }^{29}$ and later revisited by Weislogel and co-workers, ${ }^{21}$ notably in the case of a rounded corner, has shown that the aforementioned diver-

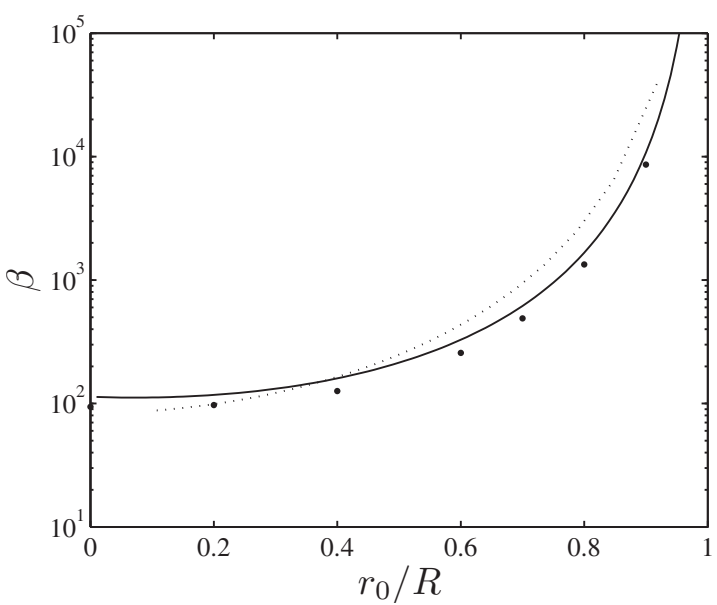

FIG. 4. Hydraulic resistance $\beta$ as a function of the tube degree of roundedness $r_{0} / R$ for a $\pi / 2$ corner angle, a perfectly wetting liquid and a free boundary condition at the liquid-air interface. Dots: results of Ransohoff and Radke (Ref. 20); solid line: results of Zhou et al. (Ref. 14); dashed line: results of Chen et al. (Ref. 21) calculated using the formulas given in Appendix A, with $r_{0}^{*}=0.1$.

gence of the hydraulic resistance disappears when using a proper scaling of the problem. However, as mentioned by Chen et al. in their paper, Ransohoff and Radke's approach remains more widespread. In the present study, the use of this later approach is motivated by the close relation of the present work with the study of evaporation in capillary porous media, in which the $\beta$ formalism is often used ${ }^{6,7}$ and is coherent with previous works of some of the authors. ${ }^{7,9}$ Figure 4 shows the values of $\beta$ as a function of $r_{0} / R$ obtained from these three works (the way to get the value of $\beta$, as defined in the present paper, from the approach of Chen et $a l .{ }^{21}$ is detailed in Appendix A). The rough shape of the various curves is similar. Notably, they all diverge when $R \rightarrow r_{0}$ i.e., when the film thickness tends toward zero. However, it is worth noting that there are up to a $20 \%$ difference in $\beta$ values predicted by these three different approaches for the whole range of $r_{0} / R$ values.

As in Sec. III, liquid pressure and curvature radius are linked by Laplace's law (the longitudinal curvature still being neglected). Combining Eqs. (2), (6), and (7) leads to

$$
q_{\text {liq }}(z)=-\rho_{l} \frac{\lambda R^{4}}{\beta \mu_{l}}\left(\gamma R^{-2} \frac{d R}{d z}-\rho_{l} g\right) \quad \text { with } \quad 0<z<z_{0} .
$$

Due to evaporation along the films, the liquid flow rate decreases up to the film tips. As in Yiotis et al., ${ }^{6}$ the resulting vapor diffusion into the tube is taken into account in the modeling. Here, the mass transfer between the liquid film and the gas phase inside the tube is simply modeled introducing a mean vapor mass concentration $\bar{c}$, which is a spatial average of the vapor mass concentration over the tube cross section region occupied by the gas $A_{g}=d^{2}-\lambda R^{2}$, and assuming the gas mixture as being dilute such that the evaporation mass rate per unit of length inside the tube $Q_{e v}$ is given by 


$$
Q_{e v}=-2 \pi D h R \frac{c_{e}-\bar{c}}{d / 2}=-2 \pi D h R \frac{c_{e}-\bar{c}}{\chi R_{b m}},
$$

where $c_{e}$ is the equilibrium vapor mass concentration at the liquid-gas interface, $D$ the vapor molecular diffusion coefficient, and $h=4$ is a dimensionless coefficient of mass transfer provided by three-dimensional computations of the steady diffusion of vapor in the gas phase, inside a square capillary tube partially filled with a perfectly wetting and volatile liquid. ${ }^{30}$ The sensitivity of the numerical results to $h$ will be discussed in Sec. IV B. by

The diffusional mass flux in the gas phase $q_{\text {vap }}$ is given

$$
q_{\mathrm{vap}}(z)=-D A_{g} \frac{d \bar{c}}{d z} .
$$

The evaporation rates expected are such that the characteristic drying time $\tau_{\mathrm{dry}}=d\left(d z_{0} / d t\right)^{-1}$ is much larger than the characteristic diffusion time $\tau_{\text {diff }}=d^{2} / D$, so that vapor diffusion in the tube can be considered as being quasisteady. In the present experiments, the ratio $\tau_{\text {dry }} / \tau_{\text {diff }}$ is found to be $\mathrm{O}\left(10^{3}-10^{4}\right)$. Also, the characteristic time for the interface shape to reach a steady state is much smaller than $\tau_{\text {dry }}$, so that the interface shape variation can be considered as being quasisteady. ${ }^{6}$ Consequently, one obtains from mass conservation

$$
Q_{e v}=\frac{d q_{\mathrm{liq}}}{d z}=-\frac{d q_{\mathrm{vap}}}{d z} .
$$

The total mass flux is denoted as $q_{\mathrm{tot}}, q_{\mathrm{tot}}=q_{\mathrm{liq}}+q_{\mathrm{vap}}$ and is constant along $z$. Note that because the $z$-axis is pointing in the same direction as gravity in the present modeling (see Fig. 1), all the fluxes defined above have negative values.

If we focus on the moment when the film tips depin from the tube top, the two following boundary conditions have to be used:

$$
\begin{aligned}
& q_{\text {vap }}(0)=q_{\text {tot }}, \\
& q_{\text {liq }}(0)=0 .
\end{aligned}
$$

As in the previous hydrostatic analysis, the gravity force is neglected over the bulk meniscus vertical extension. In addition, it is assumed that the liquid flow rate is small enough to consider that the capillary pressure jump at the bulk meniscus is constant and equal to its static value. Also, the vapor mass concentration on the liquid-gas interface is assumed to be constant and equal to its equilibrium value. The two resulting boundary conditions reads

$$
\begin{aligned}
& R\left(z_{0}\right)=R_{b m}, \\
& \bar{c}\left(z_{0}\right)=c_{e} .
\end{aligned}
$$

The above equations are made dimensionless using the following variables:

$$
R^{*}=R / R_{b m}, \quad z^{*}=z / L, \quad c^{*}=\bar{c} / c_{e} .
$$

Combining Eqs. (8), (9), and (11), a second-order nonlinear ordinary differential equation on $R^{*}$ is obtained
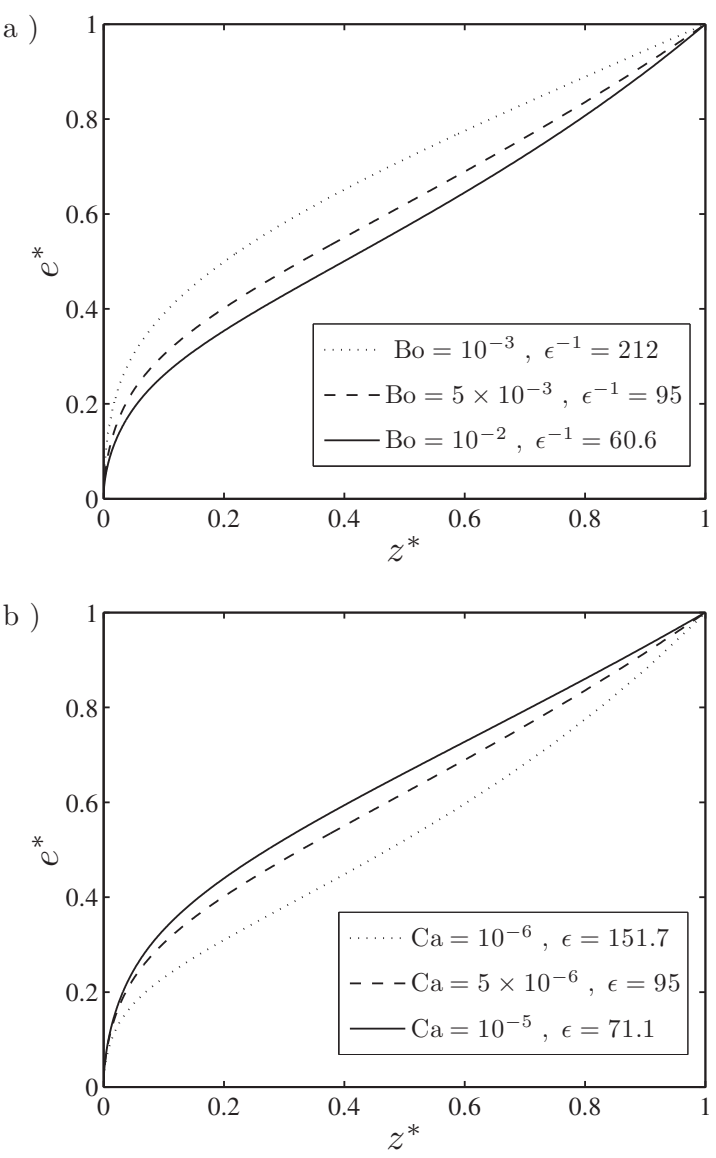

FIG. 5. Dimensionless film thickness $e^{*}=\left(R^{*}-r_{0}^{*}\right) /\left(1-r_{0}^{*}\right)$ as a function of the dimensionless $z^{*}$ coordinate. $r_{0}^{*}=0.4,\left|q_{\mathrm{tot}}^{*}\right|=4.5$, and the results of Zhou et al. were used for $\beta$. (a) $\mathrm{Ca}=5 \times 10^{-6}$. (b) $\mathrm{Bo}=5 \times 10^{-3}$.

$$
\begin{aligned}
R^{*} \frac{d^{2} R^{*}}{d z^{* 2}} & +\left(2-\frac{R^{*}}{\beta} \frac{d \beta}{d R^{*}}\right)\left(\frac{d R^{*}}{d z^{*}}\right)^{2}-\text { Bo } \epsilon^{-1} R^{* 2} \\
& \times\left(4-\frac{R^{*}}{\beta} \frac{d \beta}{d R^{*}}\right) \frac{d R^{*}}{d z^{*}}=\epsilon^{-2} \beta \text { Ca } h\left(1-c^{*}\right),
\end{aligned}
$$

where $\epsilon=R_{b m} / L$ as before and Ca is the capillary number

$$
\mathrm{Ca}=\frac{2 \pi \mu_{l} D c_{e}}{R_{b m} \rho_{l} \lambda \gamma} .
$$

Combining Eqs. (9)-(11), another second-order ordinary differential equation on $c^{*}$ is obtained,

$$
\left(4 \chi^{2}-\lambda R^{* 2}\right) \frac{d^{2} c^{*}}{d z^{* 2}}-2 \lambda R^{*} \frac{d R^{*}}{d z^{*}} \frac{d c^{*}}{d z^{*}}=-2 \pi \epsilon^{-2} h R^{*}\left(1-c^{*}\right) .
$$

The boundary conditions, Eqs. (12)-(15), become

$$
\begin{aligned}
& q_{\text {vap }}^{*}(0)=q_{\mathrm{tot}}^{*}, \\
& q_{\mathrm{liq}}^{*}(0)=0, \\
& R^{*}(1)=1,
\end{aligned}
$$




$$
c^{*}(1)=1,
$$

where the flux are made dimensionless by a reference evaporation rate $q_{\mathrm{ref}}=R_{b m} D c_{e}$ and with $q_{\mathrm{tot}}^{*}=q_{\mathrm{liq}}^{*}+q_{\mathrm{vap}}^{*}$. In order to close this set of equations the following boundary condition is added:

$$
R^{*}(0)=r_{0}^{*},
$$

traducing the drying of the film tips at depinning, the situation of interest in the present study.

The two coupled Eqs. (17) and (19), along with the corresponding boundary conditions [Eqs. (20)-(24)], form a boundary value problem with one unknown parameter $\epsilon$. An iterative scheme is used to solve this problem. Equations (17) and (19) are solved successively. At each iteration, Eq. (17) on $R^{*}$, with boundary conditions (21), (22), and (24), and Eq. (19) on $c^{*}$, with boundary conditions (20) and (23), are solved using the MATLAB solver bvp5c. The parameter $\epsilon$ is calculated in the same time as $R^{*}$. The maximal relative error on $R^{*}, c^{*}$, and $\epsilon$ is $10^{-6}$. The hydraulic resistance as a function of the curvature radius was obtained using either the results of Zhou et al. ${ }^{14}$ or Chen et al. ${ }^{21}$ which provide analytical expressions for $\beta$, as opposed to the discrete values of Ransohoff et al. ${ }^{20}$ As $\beta$ and $R^{*} \beta^{-1} d \beta / d R^{*}$ diverge when $R^{*} \rightarrow r_{0}^{*}$, the boundary condition Eq. (24) is in fact applied as $R^{*}(0)=x r_{0}^{*}$ with $x=1.001$ which, after several tests, appeared to be sufficient to obtain a converged solution. Note that in the present numerical procedure, the value of $q_{\mathrm{tot}}$ is used as an input. Three-dimensional numerical simulations of the vapor diffusion problem in the capillary tube, from the meniscus surface to the stagnant ambient air, could be used to compute the evaporation rate as a function of the bulk meniscus position and of the full meniscus surface shape. Some of the authors reported work in that direction in a recently published paper. $^{8}$ However, this numerical procedure is heavy and for simplicity, the values of $q_{\text {tot }}$ will be obtained experimentally in the present study.

\section{B. Numerical results}

In Fig. 5, the dimensionless film thickness $e^{*}=\left(R^{*}\right.$ $\left.-r_{0}^{*}\right) /\left(1-r_{0}^{*}\right)$ is shown as a function of $z^{*}$ for several values of Bond and capillary numbers. The value of $q_{\mathrm{tot}}^{*}$ used is typical of the values measured at the depinning, for the present experiments (see Table III in Sec. V). The corresponding dimensionless film length at depinning $\epsilon^{-1}=L / R_{b m}$ is given in the legend. In Fig. 5(a), film thickness profiles are shown for several Bond numbers at $\mathrm{Ca}=5$ $\times 10^{-6}$. The effect of gravity on such profiles is clearly visible: the corner film profile is all the more thin and short that the Bond number is large. In Fig. 5(b), film thickness profiles are for several capillary numbers at $\mathrm{Bo}=5 \times 10^{-3}$ for the same set of parameters as in Fig. 5(a). The viscous effects clearly limit the film length.

The effect of $r_{0}$ on the film length at depinning, the study of which is the primary objective of this paper, is displayed in Fig. 6 for different values of the capillary number at a fixed value of $\left|q_{\text {tot }}\right|$ and of the Bond number. As might have been expected from the discussion in Sec. III concerning the hydrostatic nonvolatile liquid case, it is found that the corner

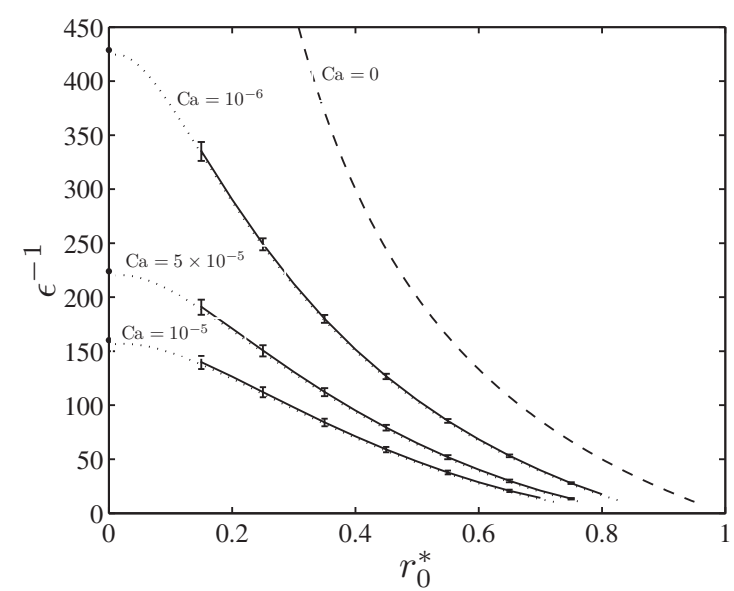

FIG. 6. Dimensionless liquid film length $\epsilon^{-1}=L / R_{b m}$ as a function of the dimensionless degree of roundedness $r_{0}^{*}$ for various Ca numbers. Solid and dashed lines correspond to the results of the complete model and the dotted lines to those of the simplified model (see Sec. IV C). The dots indicated for $r_{0}^{*}=0$ are the results given by the complete model in the case of a perfectly sharp corner. For all the curves, Bo $=5 \times 10^{-3},\left|q_{\text {tot }}^{*}\right|=4.5$, and the results of Zhou et al. were used for $\beta$.

films' maximal extension (i.e., obtained at the moment when they depin from the top of the tube) is found to decrease with $r_{0}^{*}$. This is an important effect. For instance, for $\mathrm{Ca}=10^{-6}$, the film length at depinning is divided by more than a factor of 2 between the case of a perfectly sharp corner and the case of a rounded tube with $r_{0}^{*}=0.4$ (which corresponds to the $r_{0}^{*}$ value for tube 1 , see Table I). For any value of $r_{0}^{*}$, it is important to note that the film extension is finite and much smaller that the one that is obtained in a purely hydrostatic case, when $\mathrm{Ca}=0$, which highlights how the viscous effects induced by the liquid flow toward the tube top limit the film length. When $r_{0}^{*} \rightarrow 1$, the film length at depinning decreases: as the present model is no longer valid when the film becomes so short that the effect of the longitudinal curvature on the film length cannot be neglected, no results for $\epsilon^{-1}<10$ are shown in Fig. 6. The film length depends weakly on the value of the dimensionless mass transfer coefficient $h$ [see Eq. (9)]. For instance, for $\mathrm{Ca}=10^{-5}, \mathrm{Bo}=5 \times 10^{-3}, r_{0}^{*}=0.4$, and $\left|q_{\mathrm{tot}}^{*}\right|=4.5, \epsilon^{-1}$ varies between 71.2 and 70.6 when $h$ is varied between 2 and 6 . This weak dependence of the film length on $h$ is due to the fact that the extension within the tube over which the vapor flow rate $q_{\text {vap }}$ is nonzero is always much shorter than the film length, for all the tested values of $h$ (see Fig. 7 below). The sensitivity of the obtained film length to the fixed value of $q_{\text {tot }}$ chosen for the computations at a given $\mathrm{Ca}$ and $r_{0}^{*}$ is also shown in Fig. 6. The results obtained for $\left|q_{\text {tot }}\right|=4.5 \pm 0.45$ are plotted as errors bars in Fig. 6 . This clearly displays that the film length is mainly controlled by the tube roundedness. For instance, at $\mathrm{Ca}=5$ $\times 10^{-6}$ and $r_{0}^{*}=0.3$, a $10 \%$ variation in $\left|q_{\text {tot }}\right|$ results in a variation of $\approx 3.5 \%$ for $\epsilon^{-1}$, whereas a $10 \%$ variation in $r_{0}^{*}$ results in a variation of $\approx 10 \%$ for $\epsilon^{-1}$.

In Sec. V, the predictions of the above model will be tested against experimental data. However, such a comparison is limited to the film length at depinning only, as the system of coupled equations is solved when the films depin, as expressed by boundary conditions (20) and (21). Before 
a )

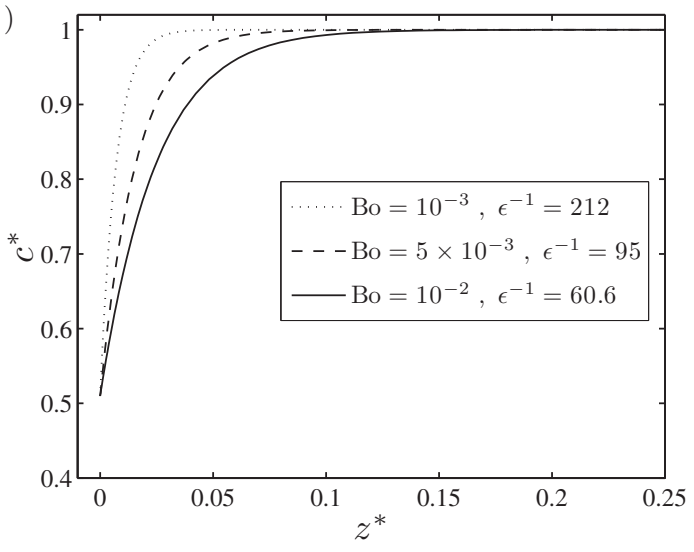

b )

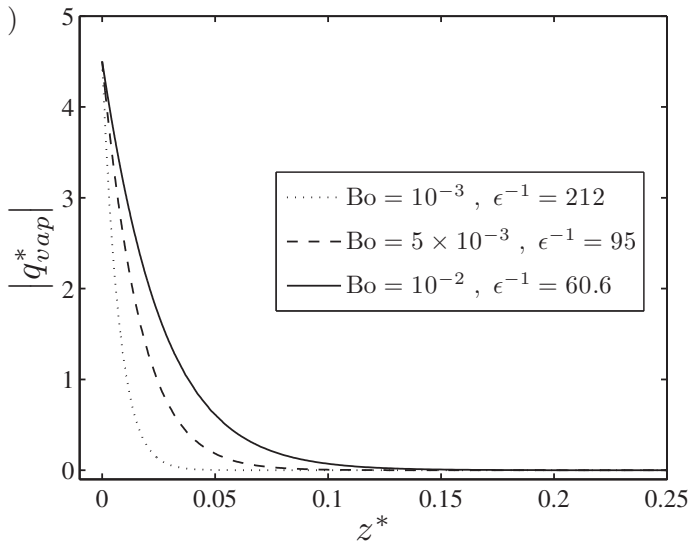

FIG. 7. (a) Dimensionless vapor mass concentration $c^{*}$ as a function of $z^{*}$. (b) Absolute value of the dimensionless vapor mass flux $q_{\text {vap }}^{*}$ as a function of $z^{*}$. These results are obtained using $\mathrm{Ca}=5 \times 10^{-6}, r_{0}^{*}=0.4,\left|q_{\mathrm{tot}}^{*}\right|=4.5$, and the results of Zhou et al. for $\beta$.

that moment, the flow rate distribution between the gas and liquid phase (i.e., the ratio between $q_{\text {liq }}$ and $q_{\text {vap }}$ ) at the film tip is not known and thus the model cannot be used to make any predictions on the film length. However, as can be seen in Fig. 7(a), where the vapor mass concentration profiles in the tube are shown for the same set of parameters as in Fig. 5(a), the gas phase is saturated over much of the film length: the vapor concentration gradients are only significant near the tube entrance region for $0<z^{*}<0.1$. Consequently, the phase change occurs preferentially at the film tip: the vapor mass flux is nonzero only in the near tube entrance region, as seen in Fig. 7(b), which shows the absolute value of the dimensionless vapor mass flux $q_{\text {vap }}^{*}$ as a function of $z^{*}$. In the following, a simpler model is proposed, in which vapor diffusion is neglected in the tube: the air is considered to be saturated in vapor inside the tube, so that $q_{\text {vap }}^{*}=0$ at any $z^{*}>0$. Consequently, the liquid flow rate is assumed to be constant along the tube. This model is similar to the one proposed in Chauvet et al. ${ }^{9}$ and it is shown below that it can predict the evolution of the film thickness during the whole experiment and not only at the moment when the films depin.

\section{A simpler model}

With the additional assumption on the saturation of air with vapor inside the tube, the problem amounts to a single differential equation, obtained by rewriting Eq. (8) as

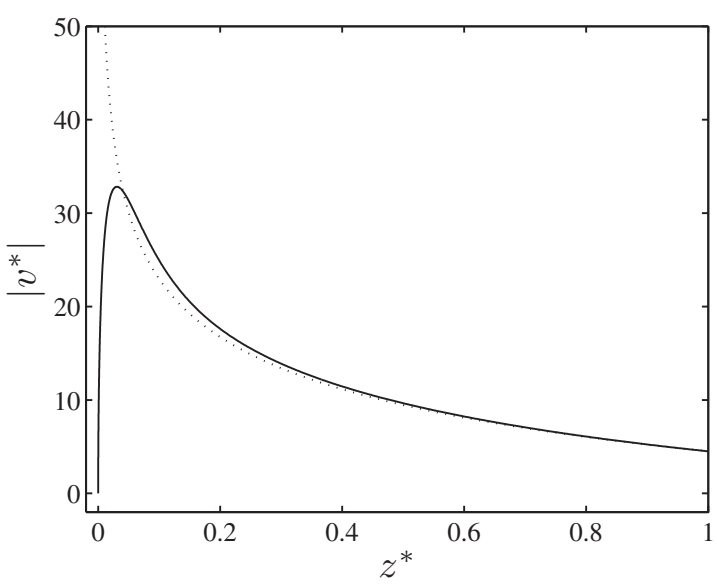

FIG. 8. Absolute value of the dimensionless liquid mean velocity $\left|v^{*}\right|$ as a function of $z^{*}$. These results are obtained using $\mathrm{Bo}=10^{-2}, \mathrm{Ca}=5 \times 10^{-6}$, $r_{0}^{*}=0.4,\left|q_{\mathrm{tot}}^{*}\right|=4.5$, and the results of Zhou et al. for $\beta$. Solid line: complete model; dotted line: simplified model.

$$
\frac{d \widetilde{z}}{d R^{*}}=\left(\frac{\mathrm{Ca}}{2 \pi} q_{\mathrm{tot}}^{*} \beta R^{*-2}+\mathrm{Bo} R^{* 2}\right)^{-1},
$$

where now $\tilde{z}=z / R_{b m}$. Using $q_{\text {tot }}$ as an input and solving Eq. (25) using a finite difference scheme with the boundary condition

$$
\widetilde{z}=0 \quad \text { at } \quad R^{*}=x r_{0}^{*} \text {, with } \quad x=1.001
$$

leads to a prediction of the film length at depinning, which is then $\epsilon^{-1}=\widetilde{z}\left(R^{*}=1\right)$. Equation (25) can also be solved using the following boundary condition:

$$
\tilde{z}=\tilde{z}_{0} \quad \text { at } \quad R^{*}=1 \text {, }
$$

using the measured $q_{\text {tot }}$ and film length as inputs in order to get the film thickness profile $R^{*}(\widetilde{z})$.

The predictions of this simple model as far as the film length at depinning is concerned, i.e., using boundary condition (26), are shown as dotted lines in Fig. 6. The simple model provides film lengths very close to the complete model predictions (solid lines in Fig. 6) for a wide range of capillary numbers, Bond numbers, and total evaporation rate values. It can be noted that the film lengths given by this simplified model are always slightly lower than those obtained with the previous approach (the maximal difference for the results presented in Fig. 6 is $2.5 R_{b m}$ for the $\mathrm{Ca}=10^{-6}$ case, at $\left.r_{0}^{*}=0.15\right)$. Indeed, in the simple model, the liquid flow rate is constant along the entire film length. Therefore, it can be argued that in this case, the liquid pressure gradient $d p_{l} / d z$, in the film tip region, is larger than the one obtained using the previous model. As $d p_{l} / d z \propto$ $-d[1 / R(z)] / d z$, the radius of curvature gradient is then lower in the previous model than in the simplified model, leading to larger film length at depinning. It is also important to notice that even if this simplified model is in good agreement with the previous one as far as film length at depinning is concerned, it predicts a divergence of the dimensionless liquid mean velocity at the film tip $v^{*}$ (see Fig. 8), where 


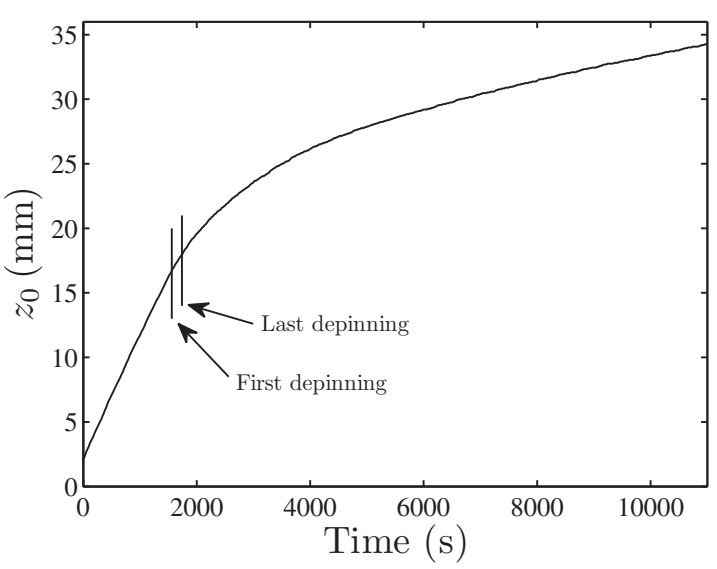

FIG. 9. Bulk meniscus evolution as a function of time for heptane in tube 2 . The moment when the first and last film depinnings are observed are indicated on the graph.

$$
v^{*}=\frac{q_{\mathrm{liq}} /\left[\rho_{l} \lambda\left(R^{2}-r_{0}^{2}\right)\right]}{q_{\mathrm{ref}} /\left[\rho_{l} \lambda\left(R_{b m}^{2}-r_{0}^{2}\right)\right]}=q_{\mathrm{liq}}^{*} \frac{1-r_{0}^{* 2}}{R^{* 2}-r_{0}^{* 2}} .
$$

Indeed, since the liquid flow rate is constant along the film and since the liquid cross section at the film tip is 0 , a divergence of $v^{*}$ is found at the film tip. On the contrary, as the complete model takes into account evaporation along the films, it predicts a more realistic zero liquid mean velocity at the film tip (see Fig. 8).

\section{EXPERIMENTAL RESULTS AND COMPARISONS WITH THE MODELS PREDICTIONS}

A typical evolution of the bulk meniscus location $z_{0}$ as a function of time is shown in Fig. 9 for heptane in tube 2 . Three distinct periods can be seen. First, as long as $z_{0}<17 \mathrm{~mm}$, the evaporation rate is roughly constant. Then, around $z_{0} \approx 17 \mathrm{~mm}$, the evaporation rate starts to decrease and this corresponds to the moment when the films depin from the tube's opened end, as can be checked visually on the recorded images. For this given experiment, the film tip depinnings do not occur exactly at the same time and the moment when the first and last film depinning occur are indicated in Fig. 9 (note that only three corner films out of four can be detected on the high magnification images, see Sec. III). Finally, after the film depinnings, the film tips recede inside the tube. Consequently, the distance between the film tips and the tubes' opened end increases, provoking an increase in the diffusive resistance to mass transfer and thus a continuous decrease of the evaporation rate, as can seen in Fig. 9 for $z_{0}>17 \mathrm{~mm}$. A more detailed analysis of this kind of curve can be found in Chauvet et al. ${ }^{8}$

We now turn to the comparison between the experimental data and the model, as far as the film length at depinning is concerned. To allow for such a comparison, the evaporation rate, made dimensionless by dividing by $q_{\mathrm{ref}}=R_{b m} D c_{e}$, has to be used as an input for the model [see boundary Eq. (20)]. The evaporation rate is estimated as

$$
\begin{aligned}
E= & \rho_{l} \frac{d z_{0}}{d t}\left\{d^{2}-(4-\pi) R_{b m}^{2}-(4-\pi) R_{b m}^{2}\right. \\
& \left.\times\left[\frac{1}{\left(1+\mathrm{Bo} \frac{z_{0}}{R_{b m}}\right)^{2}}-1\right]\right\}
\end{aligned}
$$

(see Appendix B). The $d z_{0} / d t$ value is calculated as the value at $z_{d}$ of the derivative of the best-fitting second-order polynomial of the data points $z_{0}(t)$ in the range $\left[z_{d}-1 \mathrm{~mm}\right.$, $\left.z_{d}+1 \mathrm{~mm}\right]$, where $z_{d}$ is the bulk meniscus position at the depinning. When a differential depinning of the observed films occurs, the data points $z_{0}(t)$ in the range $\left[z_{d, f}-1 \mathrm{~mm}\right.$, $\left.z_{d, l}+1 \mathrm{~mm}\right]$ are fitted by a second-order polynomial, which is then used to compute $d z_{0} / d t$ at $z_{d, f}$ and $z_{d, l}$. Here, $z_{d, f}$ and $z_{d, l}$ are the bulk meniscus positions observed at the first and last depinning. Two dimensionless evaporation rates are then calculated, $q_{\mathrm{tot}, f}^{*}$ and $q_{\mathrm{tot}, l}^{*}$, corresponding, respectively, to the evaporation rate measured when the first and last film depinnings are observed, made dimensionless by dividing by $q_{\text {ref }}$. For a given couple tube fluid, several experiments were performed, each one leading to one or two values for the dimensionless evaporation rate depending on the occurrence or not of a differential depinning. All these values were then averaged to get a unique dimensionless evaporation rate $q_{\text {tot }}^{*}$ to be used in the model, the uncertainty on this parameter being taken as the standard deviation on this average. Similarly, the film lengths at depinning were measured in each experiment, leading to one $\left(z_{d}\right)$ or two values in case of differential depinning $\left(z_{d, f}\right.$ and $\left.z_{d, l}\right)$. All these values were then averaged to get a unique film length $L_{d}$, the uncertainty on this parameter being taken as the standard deviation on this average. The values for $q_{\text {tot }}^{*}, L_{d}$, the corresponding experimental uncertainties, along with the capillary and Bond numbers calculated using the fluid properties given in Table I, are given in Table III.

Figures 10(a) and 10(b), respectively, show the predicted value of $\epsilon^{-1}=L_{d} / R_{b m}$ at depinning as a function of $r_{0}^{*}$, together with the present experimental measurements for heptane and 2-propanol, respectively. The theoretical predictions [shown as lines in Figs. 10(a) and 10(b)] are obtained using the result of Zhou et al. ${ }^{14}$ for $\beta$. For the sake of clarity, the theoretical predictions obtained using the result of Chen et $a l^{21}$ for $\beta$ are not shown. Note that they do not differ from the ones obtained using the results of Zhou et al. by more than $5.4 \%$. The vertical error bars on the theoretical predictions come from the uncertainty on $q_{\text {tot }}^{*}$. The theoretical prediction were computed only for a restricted range of $r_{0}^{*}$ around the experimental value for the tube considered. The data points are shown as isolated symbols: squares for the experiments performed with tubes 3 and 4 (having the smaller $r_{0}$ ) and circles for the experiments performed with tubes 1 and 2. The uncertainty on the degree of roundedness of the tube internal corners is shown as a horizontal error bar for the experimental data and the uncertainty on $\epsilon^{-1}$ is due to the experimental uncertainty on $L_{d}$.

The tube internal corner degree of roundedness has a huge impact on the film length at depinning. For instance, $L_{d}$ 


\begin{tabular}{lcccccc}
\hline \hline & $\mathrm{Ca}$ & $\mathrm{Bo}$ & $\left|q_{\mathrm{tot}}^{*}\right|$ & $\delta q_{\text {tot }}^{*}$ & $\begin{array}{c}L_{d} \\
(\mathrm{~mm})\end{array}$ & $\begin{array}{c}\delta L_{d} \\
(\mathrm{~mm})\end{array}$ \\
\hline Tube 1: Isopropanol & $4.52 \times 10^{-6}$ & $2.57 \times 10^{-2}$ & 3.71 & 0.21 & 11.1 & 0.8 \\
Tube 2: Isopropanol & $1.13 \times 10^{-5}$ & $4.11 \times 10^{-3}$ & 2.66 & 0.24 & 13.6 & 1.4 \\
Tube 1: Heptane & $1.32 \times 10^{-6}$ & $2.38 \times 10^{-2}$ & 4.47 & 0.12 & 11.7 & 0.05 \\
Tube 2: Heptane & $3.31 \times 10^{-6}$ & $3.81 \times 10^{-3}$ & 4.69 & 0.32 & 17.1 & 0.5 \\
Tube 3: Isopropanol & $4.74 \times 10^{-6}$ & $2.34 \times 10^{-2}$ & 3.36 & 0.23 & 21.6 & 0.65 \\
Tube 4: Isopropanol & $9.25 \times 10^{-6}$ & $6.15 \times 10^{-3}$ & 3.14 & 0.44 & 22.45 & 1.55 \\
Tube 3: Heptane & $1.39 \times 10^{-6}$ & $2.17 \times 10^{-2}$ & 3.86 & 0.68 & 28.01 & 1.27 \\
Tube 4: Heptane & $2.70 \times 10^{-6}$ & $5.70 \times 10^{-3}$ & 4.07 & 0.92 & 32.03 & 2.11 \\
\hline \hline
\end{tabular}

is roughly twice larger in tube 3 than in tube 1, which having approximately the same internal size $d$ but, respectively, with $r_{0}^{*}=0.396$ and $r_{0}^{*}=0.187$ for the two fluids used. For all the data points considered, the agreement between the experimental results and the simulation results is good: the mean difference between the data points and the theoretical predictions is $12 \%$ when using the results of Zhou et al. for $\beta$ and $12.7 \%$ when using the results of Chen et al. Thus, the present modeling captures correctly the fact that the internal roundedness of the capillary tubes is the key factor controlling the film maximal length. Another point to mention is that the differential depinning sometimes observed in the present experiments could then simply be due to slight differences in the tube corners' degrees of roundedness, the "roundest" corner depinning first.

In the following, we compare the model predictions to the experimental data, as far as the film thickness evolution before depinning is concerned. As mentioned in Sec. IV, the first model presented predicts only the film length at depinning whereas the simpler, second model can be used before the film depinning, using the evaporation rate and the film length as inputs. For a given bulk meniscus position before the film depinning, the film length is simply $L=z_{0}(t)$. When computing the evaporation rate using Eq. (29), the $d z_{0} / d t$ value is obtained by finite difference, after filtering the $z_{0}(t)$ data with a Savitzky-Golay filter. The experimental results are compared to several theoretical predictions, made at the measurement point location. As the model gives the film shape until the film thickness cancels at $z=0$, the theoretical predictions shown here do not go to zero. The film length predicted at depinning, already shown in Fig. 10, can be read in Fig. 11 as the $z_{0}$ value corresponding to the last point of a given theoretical curve. We also note that because the film thickness measurement is not performed at the tube top but at $z / d=1$ in both cases, the film thickness does not fall to zero at the film depinning, which is detected at $z=0$ on the high magnification images and the moment of which is indicated on Fig. 11. Rather, the experimental data evolution after the depinning displays the shape of the liquid film tip as it passes in front of the measurement point.

First, we compare the experimental results to the model predictions for a hydrostatic case, i.e., taking $\mathrm{Ca}=0$ in Eq. (25) [dotted line in Figs. 11(a) and 11(b)]. A good agreement is found between the model and the whole experimental data

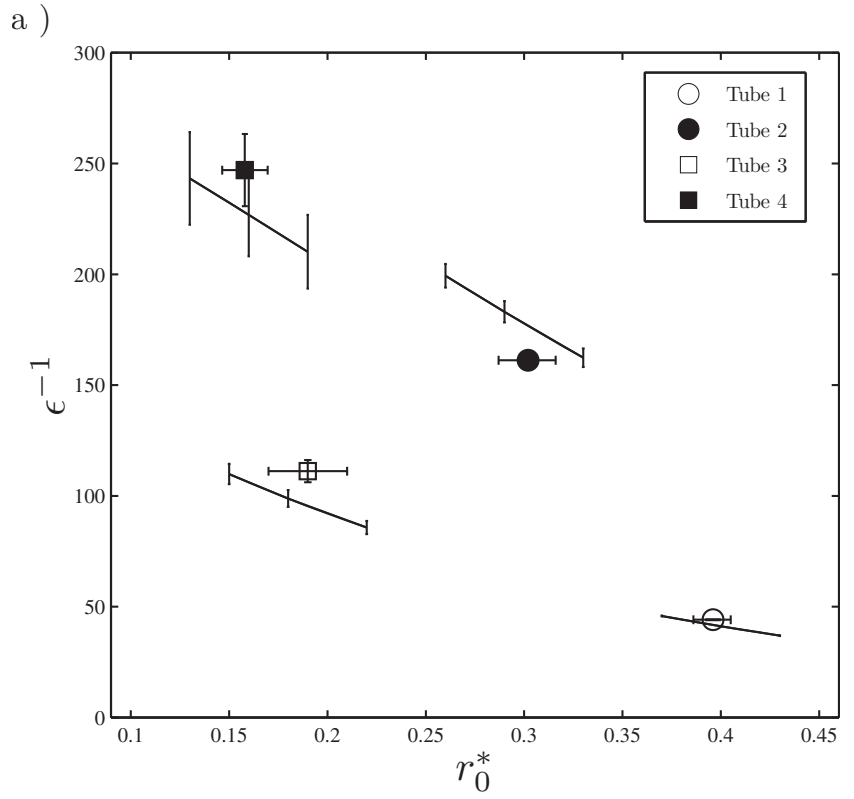

b )

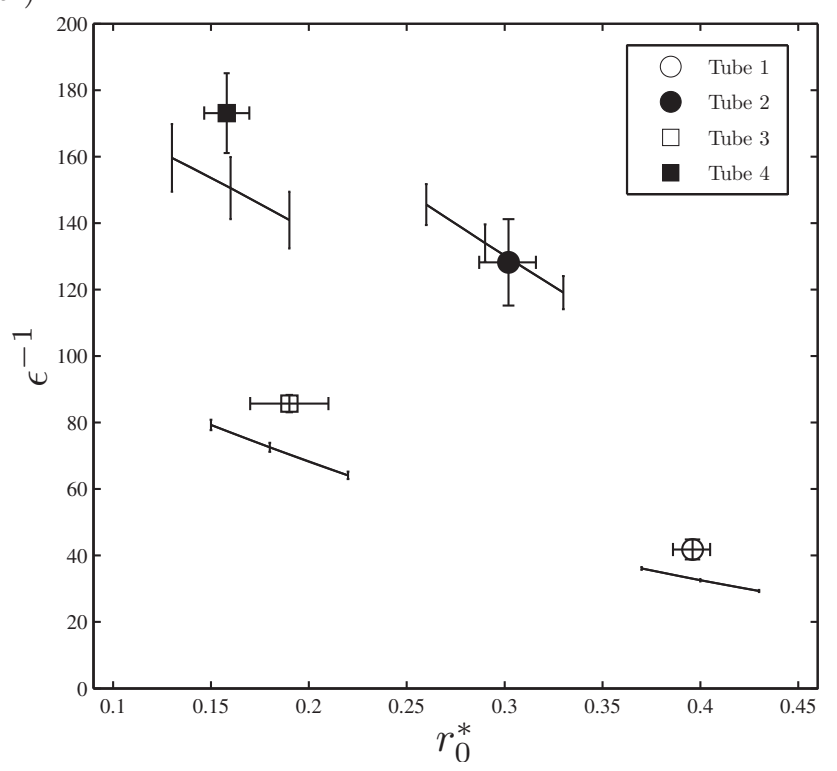

FIG. 10. Dimensionless film length at depinning $\epsilon^{-1}=L_{d} / R_{b m}$ as a function of $r_{0}^{*}$ for (a) heptane and for (b) isopropanol in the four tubes considered. The theoretical predictions are shown as solid lines for all cases for a limited range of $r_{0}^{*}$. The theoretical prediction associated with a given experimental case is the one closest to the corresponding data point. 

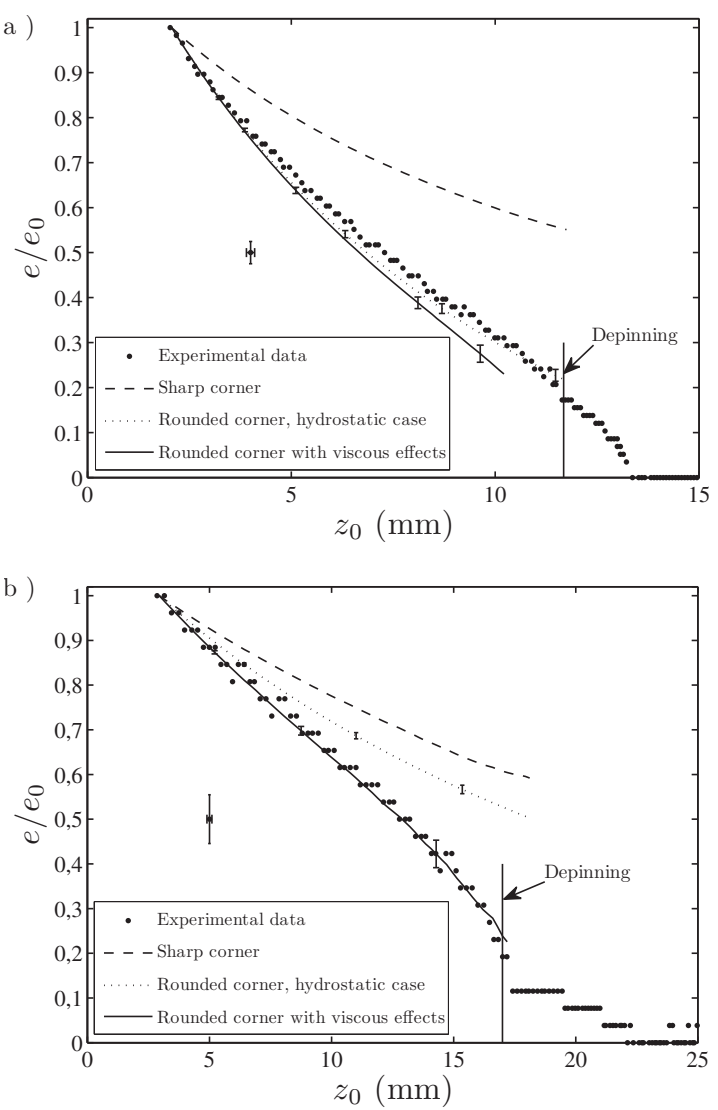

FIG. 11. Dimensionless film thickness $e / e_{0}$ as a function of $z_{0}$ for heptane in (a) tube 1 at $z=1 \mathrm{~mm}$ and (b) tube 2 at $z=0.4 \mathrm{~mm}$. The experimental error bars for the data points are shown on a distinct point, for the sake of clarity. The results of Zhou et al. were used to compute $\beta$. The vertical errors bars on the theoretical predictions come from the uncertainty on the value of $r_{0}^{*}$ used in the model.

for tube 1 , whereas in tube 2 the agreement is correct until $e / e_{0} \approx 0.8$. In tube 1 , the film thinning is then mainly due to gravity effect, whereas in the smaller tube 2, the discrepancy between a purely hydrostatic prediction and the data means that some viscous effects start to manifest when the films are getting thin enough. In this latter case, the theoretical predictions obtained when taking into account the viscous effect in the model (solid line in Fig. 11) significantly differs from the hydrostatic case [which is not the case for tube 1, see Fig. 11(a)] and agrees within errors bars with the experimental data. Note that the same conclusions can be drawn when using the results of Chen et al. for $\beta$ rather than the results of Zhou et al. To highlight the crucial importance of the internal corner degree of roundedness, we also plotted the theoretical prediction for a perfectly sharp corner, $r_{0}=0$. Then, the model clearly fails: the film thickness predicted is much larger than the one observed and also, it is not zero at the tube top, i.e., no depinning is observed.

\section{CONCLUSION}

Liquid films trapped by capillarity in the corners of a square cross section capillary tube have a great impact on the evaporation kinetics of a volatile liquid contained in the tube. They provide paths for the liquid between the receding bulk meniscus and the entrance of the tube. The capillary pump- ing of the volatile liquid from the bulk meniscus to the tube opened end where it evaporates is the key point in explaining the much faster evaporation rate obtained in a square capillary tube (compared to a circular cross section tube). In this paper, we have shown that the degree of roundedness of the tube internal corners is a key factor in controlling the corner films' maximal extension, i.e., the films' length for which the films are found to depin from the tube and to start to recede inside the tube. The tube internal roundedness provides a lower limit value for the corner films' transverse curvature and the viscous resistance to the corner flow diverges when the liquid films thin down until they perfectly match the internal corner shape. Experimental results are in good agreement with a simple model of corner flow that take into account the tube internal roundedness, as far as the maximal films' length and also the films' thickness evolution before the films' depinning are concerned.

This study highlights how a seemingly insignificant geometrical detail, the tube corners' roundedness, has a great influence on the evaporation of a volatile liquid contained in a square capillary tube. From here, one can appreciate how challenging the prediction of evaporation rate for a porous medium must be, where a precise knowledge of the pore space geometry is often out of reach experimentally and also difficult to model. Corner roundedness will certainly have a dramatic influence for others corner flow situations, such as the one typically encountered when describing microheat pipes. For instance, the dry out conditions (location and critical heat flux) should be influenced by the change in the viscous resistance to the flow into the wedge, when a slight roundedness of the wedge corner is present.

\section{ACKNOWLEDGMENTS}

Financial support from GIP ANR "Intensifilm" (Project No. ANR-06-BLAN-0119-01) is gratefully acknowledged.

\section{APPENDIX A: EXPRESSION OBTAINED FOR THE HYDRAULIC RESISTANCE $\beta$ USING THE RESULTS OF CHEN et al.}

In this short appendix, how to get the hydraulic resistance expressed using the $\beta$ formalism of Ransohoff and Radke $^{20}$ from the results Chen et al. ${ }^{21}$ is shown explicitly. Using the notations and the results of Chen et al., the hydraulic resistance $\beta$ can be expressed as ${ }^{31}$

$$
\beta=\frac{f^{2}}{F_{i}} \frac{1+T_{c}^{2}}{\left(1-r_{0}^{*}\right)^{2} T_{c}^{2}}\left\{1-\left[\frac{r_{0}^{*}}{h^{*}\left(1-r_{0}^{*}\right)+r_{0}^{*}}\right]^{2}\right\}^{-1},
$$

where $f=\sin \alpha /(\cos \alpha-\sin \alpha), \quad T_{c}=\tan \alpha+f \delta r_{0}^{*} /\left(1-r_{0}^{*}\right), \quad \delta$ $=\pi / 2-\alpha-\theta$, and $h^{*}=\left(R-r_{0}\right) /\left(R_{b m}-r_{0}\right)$. The variable $F_{i}$ is given by

$$
F_{i}=W \times F\left(\alpha, \theta, h^{*}, r_{0}^{*}\right),
$$

where the function $F$ can be found in Chen et al. and $W$ is a coefficient which depends on $h^{*}$ and $r_{0}^{*}$. The values of $W$ used to compute $\beta$ as a function of $r_{0}^{*}$ were obtained from Fig. 11 of Chen et al. for $h^{*}=1 / 2$. 


\section{APPENDIX B: DERIVATION OF AN APPROXIMATED EXPRESSION FOR THE EVAPORATION RATE}

In this appendix, the calculation leading to Eq. (29) is detailed. The evaporation rate (positive and expressed in $\mathrm{kg} \mathrm{s}^{-1}$ ) is defined by

$$
E=-\rho_{l} \frac{d\left(V_{r}+V_{c}\right)}{d t},
$$

where $V_{r}=\left[H-z_{0}(t)\right] d^{2}$ is the volume of liquid contained in the fully saturated part of the tube of vertical extension $H$ and $V_{c}=\int_{0}^{z_{0}(t)}(4-\pi) R^{2}\left[z, z_{0}(t)\right] d z$ is the volume of liquid trapped in the corners films (note that the present analysis leads to identical results when the interior corners roundedness, which is neglected here, is taken into account). Then,

$$
\frac{d\left(V_{r}+V_{c}\right)}{d t}=-d^{2} \frac{d z_{0}}{d t}+(4-\pi) \frac{d}{d t} \int_{0}^{z_{0}(t)} R^{2}\left[z, z_{0}(t)\right] d z,
$$

with

$$
\begin{aligned}
\frac{d}{d t} \int_{0}^{z_{0}(t)} R^{2}\left[z, z_{0}(t)\right] d z= & \frac{d z_{0}}{d t} R^{2}\left[z=z_{0}(t), z_{0}(t)\right] \\
& +\int_{0}^{z_{0}(t)} \frac{d}{d t} R^{2}\left[z, z_{0}(t)\right] d z,
\end{aligned}
$$

so that finally, using $R\left[z=z_{0}(t), z_{0}(t)\right]=R_{b m}$,

$$
\begin{aligned}
E= & -\rho_{l} \frac{d\left(V_{r}+V_{c}\right)}{d t} \\
= & \rho_{l} \frac{d z_{0}}{d t}\left[d^{2}-(4-\pi) R_{b m}^{2}-(4-\pi)\right. \\
& \left.\times \int_{0}^{z_{0}(t)} \frac{d}{d z_{0}} R^{2}\left[z, z_{0}(t)\right] d z\right] .
\end{aligned}
$$

The integral appearing in Eq. (B4) cannot be computed explicitly without knowledge of the shape of corner films, i.e., of $R\left[z, z_{0}(t)\right]$. Assuming that the corner films' shape is simply given by a balance between gravity and capillary forces (i.e., neglecting viscous effects),

$$
\frac{1}{R\left[z, z_{0}(t)\right]}=\frac{1}{R_{b m}}-\frac{\rho_{l} g}{\gamma}\left[z-z_{0}(t)\right]
$$

and finally,

$$
\begin{aligned}
E= & \rho_{l} \frac{d z_{0}}{d t}\left\{d^{2}-(4-\pi) R_{b m}^{2}-(4-\pi) R_{b m}^{2}\right. \\
& \left.\times\left[\frac{1}{\left(1+\mathrm{Bo} \frac{z_{0}}{R_{b m}}\right)^{2}}-1\right]\right\} .
\end{aligned}
$$

The third term appearing in Eq. (B6) becomes significant compared to the second one when the corner films are sufficiently elongated, i.e., when Bo $z_{0} / R_{b m}$ is no longer $\ll 1$. For instance, at the end of a typical experiment considered in this paper using tube 1 with isopropanol, $z_{0} / d$ is $\approx 10$ close to depinning so that Bo $z_{0} / R_{b m} \approx 1$.

Equation (B6) for $E$ is a good approximation for tubes 1 and 3, which are large enough so that viscous effects are negligible (cf. Fig. 11 and related discussion). As far as the smaller tubes used in this study are concerned (tubes 2 and 4 ), the use of Eq. (B5) to describe $R\left[z, z_{0}(t)\right]$ is more questionable as viscous effects are clearly displayed experimentally for these tubes (cf. Fig. 11). Consequently, the meniscus shape results from a balance between capillary, gravitary, and viscous effects and cannot be computed explicitly. However, as the $\mathrm{Ca}$ numbers are very small in the present study (because of the small evaporation rates), it may be expected that using Eq. (B6) should provide a precise enough estimate of the evaporation rate in the small tube case. Also, it must be noted that the two last terms in the right-hand side of Eq. (B6), and notably the problematic third one, always represent a small correction to the first one, as $(4-\pi) R_{b m}^{2} \approx 0.06 d^{2}$.

${ }^{1}$ C. B. Sobhan, R. L. Rag, and G. P. Peterson, "A review and comparative study of the investigations on micro heat pipes," Int. J. Energy Res. 31, 664 (2007).

${ }^{2}$ M. Prat, "Recent advances in pore-scale models for drying of porous media," Chem. Eng. Sci. 86, 153 (2002).

${ }^{3}$ V. S. Ajaev and G. M. Homsy, "Modeling shapes and dynamics of confined bubbles," Annu. Rev. Fluid Mech. 38, 277 (2006).

${ }^{4}$ T. M. Squires and S. R. Quakes, "Microfluidics: Fluid physics at the nanoliter scale," Rev. Mod. Phys. 77, 977 (2005).

${ }^{5}$ J. B. Laurindo and M. Prat, "Numerical and experimental network study of evaporation in capillary porous media. Drying rates," Chem. Eng. Sci. 53, 2257 (1998).

${ }^{6}$ A. G. Yiotis, A. G. Boudouvis, A. K. Stubos, I. N. Tsimpanogiannis, and Y. C. Yortsos, "The effect of liquid films on the drying of porous media," AIChE J. 50, 2721 (2004)

${ }^{7}$ M. Prat, "On the influence of pore shape, contact angle and film flows on drying of capillary porous media,” Int. J. Heat Mass Transfer 50, 1455 (2007).

${ }^{8}$ F. Chauvet, P. Duru, S. Geoffroy, and M. Prat, "Three periods of drying of a single square capillary tube," Phys. Rev. Lett. 103, 124502 (2009).

${ }^{9}$ F. Chauvet, S. Cazin, P. Duru, and M. Prat, "Use of infrared thermography for the study of evaporation in a square capillary tube," Int. J. Heat Mass Transfer 53, 1808 (2010).

${ }^{10}$ J. Stefan, "Uber das gleichgewicht und die bewegung in besondere die diffusion von gasgemengen," Sitzungsber. Akad. Wiss. Wien, Math.Naturwiss. Kl., Abt. 2A 63, 63 (1871).

${ }^{11}$ R. B. Bird, W. E. Stewart, and E. N. Lightfoot, Transport Phenomena (Wiley, New York, 2002).

${ }^{12} \mathrm{P}$. Concus and R. Finn, "On the behavior of a capillary surface in a wedge," Proc. Natl. Acad. Sci. U.S.A. 63, 292 (1969).

${ }^{13}$ Y. Pomeau, "Wetting in a corner and related questions," J. Colloid Interface Sci. 113, 5 (1986).

${ }^{14}$ D. Zhou, M. Blunt, and F. Orr, "Hydrocarbon drainage along corners of noncircular capillaries,” J. Colloid Interface Sci. 187, 11 (1997).

${ }^{15}$ M. Dong and I. Chatzis, "The imbibition and flow of a wetting liquid along the corners of a square capillary tube,” J. Colloid Interface Sci. 172, 278 (1995).

${ }^{16}$ B. Suman, S. De, and S. DasGupta, "A model of the capillary limit of a micro heat pipe and prediction of the dry-out length," Int. J. Heat Fluid Flow 26, 495 (2005).

${ }^{17}$ L. Yang and G. M. Homsy, "Steady three-dimensional thermocapillary flows and dryout inside a v-shaped wedge," Phys. Fluids 18, 042107 (2006).

${ }^{18}$ M. Markos, V. S. Ajaev, and G. M. Homsy, "Steady flow and evaporation of a volatile liquid in a wedge," Phys. Fluids 18, 092102 (2006).

${ }^{19}$ R. Savino and D. Paterna, "Marangoni effect and heat pipe dry-out," Phys. Fluids 18, 118103 (2006).

${ }^{20} \mathrm{~T}$. Ransohoff and C. Radke, "Laminar flow of wetting liquid along the corners of a predominantly gas-occupied noncircular pore," J. Colloid Interface Sci. 121, 392 (1988). 
${ }^{21}$ Y. Chen, M. M. Weislogel, and C. L. Nardin, "Capillary-driven flows along rounded interior corners," J. Fluid Mech. 566, 235 (2006).

${ }^{22} \mathrm{P}$. Concus and R. Finn, "Dichotomous behavior of capillary surfaces in zero gravity," Microgravity Sci. Technol. 3, 87 (1990).

${ }^{23}$ F. Chauvet, P. Duru, and M. Prat, "Evaporation in square capillary tubes: Optical measurement of the corner film thickness," in Proceedings of the Seventh World Conference on Experimental Heat Transfer, Fluid Mechanics and Thermodynamics (ExHFT-7 2009), edited by J. S. Szmyd, J. Spalek, and T. A. Kowalewski (AGH University of Science and Technology Press, Krakow, 2009), pp. 2007-2014.

${ }^{24}$ F. Chauvet, "Effets des films liquides en évaporation," Ph.D. thesis, Institut National Polytechnique de Toulouse, 2009.

${ }^{25} \mathrm{G}$. Mason and N. Morrow, "Meniscus curvatures in capillaries of uniform cross-section,” J. Chem. Soc., Faraday Trans. 1 80, 2375 (1984).
${ }^{26} \mathrm{H}$. Wong, S. Morris, and C. J. Radke, "Three-dimensional menisci in polygonal capillaries," J. Colloid Interface Sci. 148, 17 (1992).

${ }^{27}$ J. Bico and D. Quéré, "Rise of liquids and bubbles in angular capillary tubes," J. Colloid Interface Sci. 247, 162 (2002).

${ }^{28}$ A. Lopez de Ramos and R. L. Cerro, "Liquid filaments rise in corners of square capillaries: A novel method for the measurement of small contact angles," Chem. Eng. Sci. 49, 2395 (1994).

${ }^{29}$ P. S. Ayyaswamy, I. Catton, and D. K. Edwards, "Capillary flow in triangular grooves," ASME Trans. J. Appl. Mech. 41, 332 (1974).

${ }^{30}$ B. Camassel, N. Sghaier, M. Prat, and S. Ben Nasrallah, "Ions transport during evaporation in capillary tubes of polygonal cross section," Chem. Eng. Sci. 60, 815 (2005).

${ }^{31}$ Y. Chen (private communication). 